\title{
Equiradial designs under changing axial distances, design sizes and varying center runs with their relationships to the central composite designs
}

\author{
Iwundu, M. P *, Onu, O. H \\ ${ }^{1}$ Department of Mathematics and Statistics, University of Port Harcourt, Nigeria \\ *Corresponding author E-mail: mary.iwundu@uniport.edu.ng
}

\begin{abstract}
In assessing the preferences of equiradial designs based on design size, axial distance and number of center points in relation to the central composite designs, D-absolute deviation (D-AD) and G-absolute deviation (G-AD) are proposed as new measures of similarity of experimental designs. Each absolute deviation is positive or zero. The G-absolute deviation is zero or approximately zero at $n_{c}$ equals 1 center point. For $n_{c}$ greater than $1, \mathrm{G}$-absolute deviation decreases for increasing values of $n_{c}$. On the other hand, the D-absolute deviation decreases as the design size increases. Designs having G-AD values of zero or approximately zero are identical or near identical in G-optimality properties. Also, designs having D-AD values of zero or approximately zero are identical or near identical in D-optimality properties. It is conjecturally hoped that at some increased design size, the equiradial design and the central composite design, having same axial or radial distance will coincide (be identical) in their properties, with D-AD value of zero or approximately zero.
\end{abstract}

Keywords: Equiradial Design; Central Composite Design; Axial Distance; Design Size; Center Point; D-Absolute Deviation; G-Absolute Deviation.

\section{Introduction}

Design performance may be greatly influenced by the design region, the model type, the positioning of the design points, missing design points and a number of several other factors. Varying design regions have been considered in optimal design theory and include spherical, cuboidal, simplex and even irregular regions (See e.g. Atkinson and Donev [3]). Each design has been related to a particular model type and the composition of design points is generally a factor of some axial distance and the axial distance specifies the nature of the design geometry. Three commonly encountered axial distances have been explored in Iwundu [5]. Antille and Weinberg [2] employed perturbations of design points to study the conjecture that the less central a design point is the greater its influence on D-efficiency is. Robustness of an experimental design to one or more missing runs has been studied by Akram [1], Srisuradetchai [14] and Smucker et al. [13] as an important characteristic of experimental design. Yakubu et al. [15] considered the effects of missing observations on predictive capability of central composite designs. Kinai [10] examined the loss in efficiency when D-optimal designs are used instead of Ds optimal designs for data with missing observations. Iwundu [6] considered the behavior of alternative second-order $\mathrm{N}$-point equiradial designs under variations of model parameters for design radius $\rho=1.0$ and established relationships among some alphabetic optimality criteria with regards to the designs and the models. Iwundu and Jaja [7] considered the precision of employing full polynomial response surface designs on models with missing coefficients (reduced models) using efficiency measures. The loss in D- and G-efficiency of constructed first- and second-order exact designs were established for models with missing interaction coefficients.
As defined by Khuri and Cornel [9], an equiradial design consists of two or more sets of points where the design points for each set have the same distance from the design center. For a twodimensional design region, an equiradial design comprises of a set of five points on the circle of radius $\rho \geq 1$ from the design center. The center point (or points) forms a second set in a circle of radius zero. The rotatable central composite design due to Box and Wilson [4] is a member of the larger class of equiradial designs. For example, with $\mathrm{k}=2$ and $\alpha=\sqrt{2}$, the four factorial points and the four axial points form a set of eight points on the circle of radius $\rho=\sqrt{2}$ and the center point (or points) forms a second set on a circle of radius zero. Hence, the inscribed central composite design and the circumscribed central composite design automatically belong to the larger class of equiradial designs. Obviously, two or more equiradial sets are needed to provide a design for fitting a second-order model. The second-order equiradial designs that are rotatable include those equiradial set of points that are equally spaced on a circle, a sphere or a hypersphere and which form the vertices of a regular polygon, polyhedron or polytope. Alphabetic optimality criteria and Design efficiency have been employed in most comparative studies involving experimental designs. We shall in this work examine the equiradial designs for second-order model defined on design regions under changing axial distances, varying design sizes and varying center runs. In addition, the behavior of the designs shall be compared with central composite designs on similar design regions.

\section{Methodology}

We commence our investigation with 6-point equiradial design $\xi_{6}$ whose set of five equidistant design points consists of the equally spaced points, supported on the vertices of a regular pentagon and 
the second set of equidistant design points consists of $n_{c} \geq 1$ center points. The design $\xi_{6}$ and its design matrix, $X$, are as expressed in Khuri and Cornel [9], Myers et al. [11] and Iwundu [6]. The 6-point equiradial design with $\rho=1.0$ and a center point is

$\xi_{6}=\left(\begin{array}{cc}1 & 0 \\ 0.31 & 0.95 \\ -0.81 & 0.59 \\ -0.81 & -0.59 \\ 0.31 & -0.95 \\ 0 & 0\end{array}\right)$

The design measures associated with the equiradial designs are such that the design matrices are expressed as

$\begin{array}{cc}\mathrm{x}_{1} & \mathrm{x}_{2} \\ \left\{\rho \operatorname{Cos}\left(\theta+2 \pi u / \mathrm{n}_{1}\right)\right. & \left.\rho \operatorname{Sin}\left(\theta+2 \pi u / \mathrm{n}_{1}\right)\right\} \\ \mathrm{u}=0,1,2, \ldots, \mathrm{n}_{1}-1 & \end{array}$

where $x_{1}$ and $x_{2}$ represent the two controllable variables, $\rho$ is the radius of the design, $n_{1}$ represents the number of points on the sphere and $\mathrm{n}_{\mathrm{c}}$ represents the number of center points. This standard expression shall be employed in obtaining the design measures for the required N-point equiradial designs. Following Myers et al. [11], the value of $\theta$ is assumed equal to zero since $\theta$ has no effect on the information matrix, $\mathrm{X}^{\mathrm{T}} \mathrm{X}$, of the design. For the design matrix, $X$, the associated information matrix, $X^{\prime} X$, is assumed nonsingular and normalized as $\frac{X^{\prime} X}{N}=M$, where $N$ is the design size. For the purpose of this research, the non-center points $\mathrm{n}_{1}$, called the radial points, shall lie between 5 and 8 , inclusive. In addition to the $n_{1}$ radial points of the design, $1 \leq n_{c} \leq 5$ center points shall be included in the design. Thus, the N-point design shall comprise of a set of $n_{1}$ radial points and $n_{c}$ center points. There exists the inverse matrix $\mathrm{M}^{-1}$ which is symmetric and represents a matrix of estimates of variances of parameters and covariances among parameters. The inverse matrix is cardinal when comparing designs as most optimality criteria, as in Rady et al [12], are defined as functional of $\mathrm{M}^{-1}$. In establishing the preferences of the equiradial designs over the central composite designs under changing axial distances, design sizes and varying center runs, we shall concern ourselves with the D- and G-efficiency. These criteria have been well explained and illustrated in a good number of literatures on optimal design of experiments with recent works offering modifications to suit experimental conditions. An example of such documentations is due to Srisuradetchai [14]. The determinant of information matrix as well as the scaled predictive variances associated with each design shall be computed. Two axial distances $\alpha=1.0$ and $\alpha=1.414$ shall define the geometry of the design region. Specifically, the equidistant points (different from the center points) of the equiradial designs shall make radius of $\rho=1.0$ or $\rho=1.414$ thus resulting in axial distance of $\alpha=1.0$ or $\alpha=1.414$, respectively. The rotatable central composite design shall be defined for $\alpha=1.414$. Such rotatable central composite design is called circumscribed central composite design and comprises of the design points $(-1,-1),(1,-1),(-1,1),(1,1),(\alpha, 0)$, $(-\alpha, 0),(0, \alpha),(0,-\alpha)$ and $\mathrm{n}_{\mathrm{c}}$ centre points $(0,0)$, where $\alpha \cong 1.414$. The inscribed central composite design also uses $\alpha$ value of 1.414 to describe a circular geometric region. However, the inscribed central composite design restricts the actual design region to the defined variable ranges \pm 1 by locating the axial points at the lower and upper bounds of the variable ranges. Thus, the factorial points are brought into (inscribed into) the interior of the design space. This way, $\alpha$ value becomes 1.0. Unlike the rotatable circumscribed central composite design which uses design points ranging between -1.414 and +1.414 , the rotatable inscribed central composite design uses design points ranging between -1 and +1 and comprises of the design points $(-0.7,-0.7),(0.7,-0.7),(-0.7,0.7)$, $(0.7,0.7),(1,0),(-1,0),(0,1),(0,-1)$ and $\mathrm{n}_{\mathrm{c}}$ centre points $(0,0)$. These design points shall make up the N-point exact design compositions. Iwundu and Otaru [8] presented the composition of $\mathrm{N}$ - point exact designs for each of the inscribed and circumscribed central composite designs. The 6-point circumscribed central composite design contains the design points $(-1,-1),(1,-1)$, $(-1,1),(1,1),(1,0),(0,0)$. The 7 -point circumscribed central composite design contains the design points $(-1,-1),(1,-1)$, $(-1,1),(1,1),(1,0),(0,-1),(0,0)$. The 8-point circumscribed central composite design contains the design points $(-1,-1)$, $(1,-1),(-1,1),(1,1),(1,0),(-1,0),(0,1),(0,0)$. The 9-point circumscribed central composite design contains the design points $(-1,-1),(1,-1),(-1,1),(1,1),(1,0),(-1,0),(0,1),(0,-1),(0,0)$. The 6-point inscribed central composite design contains the design points $(-0.7,0.7),(0.7,0.7),(0,-1),(-1,0),(1,0),(0,0)$. The $7-$ point inscribed central composite design contains the design points $(-0.7,0.7),(0.7,0.7),(-0.7,-0.7),(0,-1),(-1,0),(1,0)$, $(0,0)$. The 8 -point inscribed central composite design contains the design points $(-0.7,0.7),(0.7,0.7),(-0.7,-0.7),(0.7,-0.7),(0,-1)$, $(-1,0),(1,0),(0,0)$. The 9-point inscribed central composite design contains the design points $(-0.7,0.7),(0.7,0.7),(-0.7,-0.7)$, $(0.7,-0.7),(0,-1),(0,1),(-1,0),(1,0),(0,0)$.

With these arrangements, we shall (i) Examine the behaviour of the spherical equiradial designs for changing axial distances. (ii) Examine the behaviour of the spherical equiradial designs for changing design sizes. (iii) Examine the behaviour of the spherical equiradial designs for increased center points. (iv) Examine the behaviour of the spherical central composite designs for changing axial distances. (v) Examine the behaviour of the spherical central composite designs for changing design sizes. (vi) Examine the behaviour of the spherical central composite designs for increased center points. (vii) Compare the spherical equiradial designs and the spherical central composite designs on the basis of D- and G- efficiencies. (viii) Propose new measures of similarity of experimental designs. A simple layout comprising the radial points $\left(\mathrm{n}_{1}\right)$, the center points $\left(\mathrm{n}_{\mathrm{c}}\right)$, the design size $(\mathrm{N})$, the determinant of information matrix (det $(\mathrm{M}))$, the maximum scaled predictive variance $\left(\mathrm{SPV}_{\max }\right)$, the D-efficiency $\left(\mathrm{D}_{\text {eff }}\right)$ and the G-efficiency $\left(\mathrm{G}_{\text {eff }}\right)$ shall be presented as summary of computations. Each layout component has been well explained in Myers et al. [11], which serves as a major reference in Response Surface Methodology.

\section{Results}

\subsection{Spherical equiradial designs $\left(\rho=1.0, n_{c} \geq 1\right)$}

The computations involving equiradial designs constructed using $\boldsymbol{\rho}=1.0$ and $\mathrm{n}_{\mathrm{c}} \geq 1$ are as in Table 1 .

\subsection{Spherical equiradial designs $\left(\rho=1.414, n_{c} \geq 1\right)$}

The computations involving equiradial designs constructed using $\boldsymbol{\rho}=1.414$ and $n_{c} \geq 1$ are as in Table 2 .

\subsection{Circumscribed central composite designs $(\alpha=1.414$, $\left.\mathbf{n}_{\mathrm{c}} \geq \mathbf{1}\right)$}

The computations involving Circumscribed Central Composite designs constructed using $\alpha=1.414$ and $\boldsymbol{n}_{\boldsymbol{c}} \geq 1$ are as in Table 3 .

\subsection{Inscribed central composite designs $\left(\alpha=1.0, n_{c} \geq 1\right)$}

The computations involving Inscribed Central Composite designs constructed using $\alpha=1.0$ and $\boldsymbol{n}_{\boldsymbol{c}} \geq 1$ are as in Table 4 .

\subsection{D-absolute deviation and G-absolute deviation}

An absolute deviation is a measure of variability or variation. In particular, each calculated distance between any two points is called an absolute deviation. In the context of design efficiency, we shall refer to the absolute deviation of two D-efficiency values 
Table 1: Summary of Computations for Spherical Equiradial designs $\left(\boldsymbol{\rho}=1.0, \mathrm{~N}_{\mathrm{C}} \geq 1\right)$

\begin{tabular}{|c|c|c|c|c|c|c|}
\hline $\begin{array}{l}\text { Design size } \\
N\end{array}$ & $\begin{array}{l}\text { Radial point } \\
\mathrm{n}_{1}\end{array}$ & $\begin{array}{l}\text { Center point } \\
\mathrm{n}_{\mathrm{c}}\end{array}$ & $\begin{array}{l}\text { Determinant of infor- } \\
\text { mation matrix } \operatorname{det}(\mathrm{M})\end{array}$ & $\begin{array}{l}\text { Maximum scaled } \\
\text { predictive variance } \\
\text { SPV }_{\max }\end{array}$ & $\begin{array}{l}\text { D-efficiency } \mathrm{D}_{\text {eff }} \\
\%\end{array}$ & $\begin{array}{l}\text { G-efficiency } \mathrm{G}_{\text {eff }} \\
\%\end{array}$ \\
\hline 6 & 5 & 1 & $2.6100 \times 10^{-4}$ & 6 & 25.28 & 100.00 \\
\hline 7 & & 2 & $2.0700 \times 10^{-4}$ & 7 & 24.32 & 85.71 \\
\hline 8 & & 3 & $1.3954 \times 10^{-4}$ & 8 & 22.77 & 75.00 \\
\hline 9 & & 4 & $9.1756 \times 10^{-5}$ & 9 & 21.24 & 66.67 \\
\hline 10 & & 5 & $6.1000 \times 10^{-5}$ & 10 & 19.84 & 60.00 \\
\hline 7 & 6 & 1 & $2.5845 \times 10^{-4}$ & 7 & 25.24 & 85.71 \\
\hline 8 & & 2 & $2.3198 \times 10^{-4}$ & 6.671 & 24.79 & 89.94 \\
\hline 9 & & 3 & $1.717 \times 10^{-4}$ & 7.505 & 23.58 & 79.95 \\
\hline 10 & & 4 & $1.2163 \times 10^{-4}$ & 8.339 & 22.26 & 71.95 \\
\hline 11 & & 5 & $8.5819 \times 10^{-5}$ & 9.173 & 21.00 & 65.41 \\
\hline 8 & 7 & 1 & $2.5016 \times 10^{-4}$ & 8 & 25.10 & 75 \\
\hline 9 & & 2 & $2.4679 \times 10^{-4}$ & 6.492 & 25.05 & 92.33 \\
\hline 10 & & 3 & $1.9673 \times 10^{-4}$ & 7.146 & 24.12 & 83.96 \\
\hline 11 & & 4 & $1.4807 \times 10^{-4}$ & 7.861 & 23.00 & 76.33 \\
\hline 12 & & 5 & $1.0981 \times 10^{-4}$ & 8.576 & 21.88 & 69.96 \\
\hline 9 & 8 & 1 & $2.4189 \times 10^{-4}$ & 9 & 24.96 & 66.67 \\
\hline 10 & & 2 & $2.5710 \times 10^{-4}$ & 6.259 & 25.22 & 95.86 \\
\hline 11 & & 3 & $2.1769 \times 10^{-4}$ & 6.886 & 24.53 & 87.13 \\
\hline 12 & & 4 & $1.7220 \times 10^{-4}$ & 7.510 & 23.59 & 79.89 \\
\hline 13 & & 5 & $1.3316 \times 10^{-4}$ & 8.136 & 22.60 & 73.75 \\
\hline
\end{tabular}

Table 2: Summary of Computations for Spherical Equiradial designs $\left(\boldsymbol{\rho}=1.414, \boldsymbol{n}_{\boldsymbol{c}} \geq 1\right)$

\begin{tabular}{|c|c|c|c|c|c|c|}
\hline $\begin{array}{l}\text { Design size } \\
N\end{array}$ & $\begin{array}{l}\text { Radial point } \\
n_{1}\end{array}$ & $\begin{array}{l}\text { Center point } \\
n_{c}\end{array}$ & $\begin{array}{l}\text { Determinant of infor- } \\
\text { mation matrix } \operatorname{det}(M)\end{array}$ & $\begin{array}{l}\text { Maximum scaled } \\
\text { predictive variance } \\
S P V_{\max }\end{array}$ & $\begin{array}{l}\text { D-efficiency } D_{e f f} \\
\%\end{array}$ & $\begin{array}{l}\text { G-efficiency } G_{e f f} \\
\%\end{array}$ \\
\hline 6 & 5 & 1 & $6.6822 \times 10^{-2}$ & 6 & 63.70 & 100.00 \\
\hline 7 & & 2 & $5.2999 \times 10^{-2}$ & 7 & 61.29 & 85.71 \\
\hline 8 & & 3 & $3.5679 \times 10^{-2}$ & 8 & 57.38 & 75.00 \\
\hline 9 & & 4 & $2.3466 \times 10^{-2}$ & 9 & 53.51 & 66.67 \\
\hline 10 & & 5 & $1.5588 \times 10^{-2}$ & 10 & 49.98 & 60.00 \\
\hline 7 & 6 & 1 & $6.5951 \times 10^{-2}$ & 7 & 63.56 & 85.71 \\
\hline 8 & & 2 & $5.9197 \times 10^{-2}$ & 6.670 & 62.43 & 89.96 \\
\hline 9 & & 3 & $4.3800 \times 10^{-2}$ & 7.504 & 59.37 & 79.97 \\
\hline 10 & & 4 & $3.1036 \times 10^{-2}$ & 8.338 & 56.06 & 71.96 \\
\hline 11 & & 5 & $2.1899 \times 10^{-2}$ & 9.171 & 52.89 & 65.42 \\
\hline 8 & 7 & 1 & $6.4659 \times 10^{-2}$ & 7.995 & 63.35 & 75.05 \\
\hline 9 & & 2 & $6.3769 \times 10^{-2}$ & 6.498 & 63.21 & 92.34 \\
\hline 10 & & 3 & $5.0829 \times 10^{-2}$ & 7.174 & 60.86 & 83.64 \\
\hline 11 & & 4 & $3.8254 \times 10^{-2}$ & 7.941 & 58.05 & 75.56 \\
\hline 12 & & 5 & $2.8369 \times 10^{-2}$ & 8.608 & 55.23 & 69.70 \\
\hline 9 & 8 & 1 & $6.1549 \times 10^{-2}$ & 9 & 62.84 & 66.67 \\
\hline 10 & & 2 & $6.5419 \times 10^{-2}$ & 6.257 & 63.48 & 95.89 \\
\hline 11 & & 3 & $5.5391 \times 10^{-2}$ & 6.883 & 61.74 & 87.17 \\
\hline 12 & & 4 & $4.3817 \times 10^{-2}$ & 7.508 & 59.38 & 79.91 \\
\hline 13 & & 5 & $3.3883 \times 10^{-2}$ & 8.134 & 56.88 & 73.76 \\
\hline
\end{tabular}

Table 3: Summary of Computations for Circumscribed Central Composite Designs $\left(\alpha=1.414, n_{c} \geq 1\right)$

\begin{tabular}{|c|c|c|c|c|c|c|}
\hline $\begin{array}{l}\text { Design size } \\
N\end{array}$ & $\begin{array}{l}\text { Radial point } \\
n_{1}\end{array}$ & $\begin{array}{l}\text { Center point } \\
n_{c}\end{array}$ & $\begin{array}{l}\text { Determinant of infor- } \\
\text { mation matrix } \operatorname{det}(M)\end{array}$ & $\begin{array}{l}\text { Maximum scaled } \\
\text { predictive variance } \\
S P V_{\max }\end{array}$ & $\begin{array}{l}\text { D-efficiency } D_{e f f} \\
\%\end{array}$ & $\begin{array}{l}\text { G-efficiency } G_{\text {eff }} \\
\%\end{array}$ \\
\hline 6 & 5 & 1 & $3.1930 \times 10^{-2}$ & 6 & 56.32 & 100.00 \\
\hline 7 & & 2 & $2.5325 \times 10^{-2}$ & 7 & 54.19 & 85.71 \\
\hline 8 & & 3 & $1.7048 \times 10^{-2}$ & 8 & 50.73 & 75.00 \\
\hline 9 & & 4 & $1.1213 \times 10^{-2}$ & 9 & 47.31 & 66.67 \\
\hline 10 & & 5 & $7.4485 \times 10^{-3}$ & 10 & 44.19 & 60.00 \\
\hline 7 & 6 & 1 & $3.8352 \times 10^{-2}$ & 7 & 58.07 & 85.71 \\
\hline 8 & & 2 & $3.4424 \times 10^{-2}$ & 7.547 & 57.04 & 79.50 \\
\hline 9 & & 3 & $2.5470 \times 10^{-2}$ & 8.490 & 54.24 & 70.67 \\
\hline 10 & & 4 & $1.8048 \times 10^{-2}$ & 9.434 & 51.22 & 63.60 \\
\hline 11 & & 5 & $1.2735 \times 10^{-2}$ & 10.377 & 48.32 & 57.82 \\
\hline 8 & 7 & 1 & $4.6803 \times 10^{-2}$ & 8 & 60.03 & 75.00 \\
\hline 9 & & 2 & $4.6173 \times 10^{-2}$ & 7.811 & 59.90 & 76.81 \\
\hline 10 & & 3 & $3.6807 \times 10^{-2}$ & 8.679 & 57.68 & 69.13 \\
\hline 11 & & 4 & $2.7702 \times 10^{-2}$ & 9.547 & 55.01 & 62.85 \\
\hline 12 & & 5 & $2.0545 \times 10^{-2}$ & 10.415 & 52.33 & 57.61 \\
\hline 9 & 8 & 1 & $6.1548 \times 10^{-2}$ & 9 & 62.84 & 66.67 \\
\hline 10 & & 2 & $6.5418 \times 10^{-2}$ & 6.251 & 63.48 & 95.99 \\
\hline 11 & & 3 & $5.5390 \times 10^{-2}$ & 6.876 & 61.74 & 87.26 \\
\hline 12 & & 4 & $4.3817 \times 10^{-2}$ & 7.501 & 59.38 & 79.99 \\
\hline 13 & & 5 & $3.3883 \times 10^{-2}$ & 8.126 & 56.88 & 73.84 \\
\hline
\end{tabular}


Table 4: Summary of Computations For Inscribed Central Composite Designs $\left(\alpha=1.0, n_{c} \geq 1\right)$

\begin{tabular}{|c|c|c|c|c|c|c|}
\hline $\begin{array}{l}\text { Design size } \\
N\end{array}$ & $\begin{array}{l}\text { Radial point } \\
n_{1}\end{array}$ & $\begin{array}{l}\text { Center point } \\
n_{c}\end{array}$ & $\begin{array}{l}\text { Determinant of infor- } \\
\text { mation matrix } \operatorname{det}(M)\end{array}$ & $\begin{array}{l}\text { Maximum scaled } \\
\text { predictive variance } \\
S P V_{\max }\end{array}$ & $\begin{array}{l}\text { D-efficiency } D_{\text {eff }} \\
\%\end{array}$ & $\begin{array}{l}\text { G-efficiency } G_{e f f} \\
\%\end{array}$ \\
\hline 6 & 5 & 1 & $1.1660 \times 10^{-4}$ & 6 & 22.10 & 100 \\
\hline 7 & & 2 & $9.2480 \times 10^{-5}$ & 7 & 21.27 & 85.71 \\
\hline 8 & & 3 & $6.2269 \times 10^{-5}$ & 8 & 19.91 & 75.00 \\
\hline 9 & & 4 & $4.0946 \times 10^{-5}$ & 9 & 18.57 & 66.67 \\
\hline 10 & & 5 & $2.7200 \times 10^{-5}$ & 10 & 17.34 & 60.00 \\
\hline 7 & 6 & 1 & $1.3847 \times 10^{-4}$ & 7 & 22.75 & 85.71 \\
\hline 8 & & 2 & $1.2428 \times 10^{-4}$ & 7.547 & 22.34 & 79.50 \\
\hline 9 & & 3 & $9.1951 \times 10^{-5}$ & 8.490 & 21.25 & 70.67 \\
\hline 10 & & 4 & $6.5155 \times 10^{-5}$ & 9.434 & 20.06 & 63.60 \\
\hline 11 & & 5 & $4.5972 \times 10^{-5}$ & 10.377 & 18.93 & 57.82 \\
\hline 8 & 7 & 1 & $1.6676 \times 10^{-4}$ & 7.996 & 23.46 & 75.04 \\
\hline 9 & & 2 & $1.6447 \times 10^{-4}$ & 7.795 & 23.41 & 76.97 \\
\hline 10 & & 3 & $1.3110 \times 10^{-4}$ & 8.661 & 22.54 & 69.28 \\
\hline 11 & & 4 & $9.8663 \times 10^{-5}$ & 9.527 & 21.50 & 62.98 \\
\hline 12 & & 5 & $7.3168 \times 10^{-5}$ & 10.393 & 20.45 & 57.73 \\
\hline 9 & 8 & 1 & $2.2241 \times 10^{-4}$ & 8.993 & 24.61 & 66.72 \\
\hline 10 & & 2 & $2.3630 \times 10^{-4}$ & 6.301 & 24.86 & 95.22 \\
\hline 11 & & 3 & $2.0005 \times 10^{-4}$ & 6.931 & 24.18 & 86.57 \\
\hline 12 & & 4 & $1.5824 \times 10^{-4}$ & 7.561 & 23.26 & 79.35 \\
\hline 13 & & 5 & $1.2236 \times 10^{-4}$ & 8.191 & 22.28 & 73.25 \\
\hline
\end{tabular}

as D-absolute deviation (D-AD). Similarly, we shall refer to the absolute deviation of two G-efficiency values as G-absolute deviation (G-AD). Mathematically, we shall write

$\mathrm{D}-\mathrm{AD}=\left|\mathrm{D}_{\text {eff (1) }}-\mathrm{D}_{\mathrm{eff}(2)}\right| ; 0 \leq \mathrm{D}_{\mathrm{eff}(1)}, \mathrm{D}_{\mathrm{eff}(2)} \leq 1$

where

$D_{\text {eff (1) }}$ is the D-efficiency associated with design $\xi_{1}$ and $D_{\text {eff (2) }}$ is the D-efficiency associated with design $\xi_{2}$.

In a similar form, we shall write

$\mathrm{G}-\mathrm{AD}=\left|\mathrm{G}_{\text {eff (1) }}-\mathrm{G}_{\text {eff (2) }}\right| ; 0 \leq \mathrm{G}_{\text {eff (1), }} \mathrm{G}_{\text {eff (2) }} \leq 1$

where

$\mathrm{G}_{\text {eff (1) }}$ is the G-efficiency associated with design $\xi_{1}$ and $\mathrm{G}_{\text {eff (2) }}$ is the G-efficiency associated with design $\xi_{2}$. Thus, D-absolute deviation is related to D-efficiency and G-absolute deviation is related to G-efficiency.

In comparing pairs of N-point designs, $\left\{\xi_{11}, \xi_{12}\right\}$ and $\left\{\xi_{21}, \xi_{22}\right\}$, the pair of designs $\xi_{11}$ and $\xi_{12}$ are assumed more closely related in D-efficiency property than the pair of designs $\xi_{21}$ and $\xi_{22}$ if and only if

$\left|D_{\text {eff }\left(\xi_{11}\right)}-D_{\text {eff }\left(\xi_{12}\right)}\right| \leq\left|D_{\text {eff }\left(\xi_{21}\right)}-D_{\text {eff }\left(\xi_{22}\right)}\right|$.

If $\left|D_{\text {eff }\left(\xi_{11}\right)}-D_{\text {eff }\left(\xi_{12}\right)}\right|=0$, then design $\xi_{11}$ and design $\xi_{12}$ are identical in optimality property.

Also, if $\left|D_{\text {eff }\left(\xi_{21}\right)}-D_{\text {eff }\left(\xi_{22}\right)}\right|=0$, then design $\xi_{21}$ and design $\xi_{22}$ are identical in optimality property.

If $\left|D_{\text {eff }\left(\xi_{11}\right)}-D_{\text {eff }\left(\xi_{12}\right)}\right|=\left|D_{\text {eff }\left(\xi_{21}\right)}-D_{\text {eff }\left(\xi_{22}\right)}\right|$, then all four designs $\xi_{11}, \xi_{12}, \xi_{21}$ and $\xi_{22}$ are identical in optimality property.

Similarly, in comparing pairs of N-point designs, $\left\{\xi_{11}, \xi_{12}\right\}$ and $\left\{\xi_{21}, \xi_{22}\right\}$, the pair of designs $\xi_{11}$ and $\xi_{12}$ are assumed more closely related in G-efficiency property than the pair of designs $\xi_{21}$ and $\xi_{22}$ if and only if

$\left|\mathrm{G}_{\text {eff }\left(\xi_{11}\right)}-\mathrm{G}_{\text {eff }\left(\xi_{12}\right)}\right| \leq\left|\mathrm{G}_{\text {eff }\left(\xi_{21}\right)}-\mathrm{G}_{\text {eff }\left(\xi_{22}\right)}\right|$.

If $\left|G_{\text {eff }\left(\xi_{11}\right)}-G_{\text {eff }\left(\xi_{12}\right)}\right|=0$, then design $\xi_{11}$ and design $\xi_{12}$ are identical in optimality property.

Also, if $\left|G_{\text {eff }\left(\xi_{21}\right)}-G_{\text {eff }\left(\xi_{22}\right)}\right|=0$, then design $\xi_{21}$ and design $\xi_{22}$ are identical in optimality property.

If $\left|G_{\text {eff }\left(\xi_{11}\right)}-G_{\text {eff }\left(\xi_{12}\right)}\right|=\left|G_{\text {eff }\left(\xi_{21}\right)}-G_{\text {eff }\left(\xi_{22}\right)}\right|$, then all four designs $\xi_{11}, \xi_{12}, \xi_{21}$ and $\xi_{22}$ are identical in optimality property.

For the designs studied in this research work, we present the Dand G-absolute deviations in Table 5. Each absolute deviation is positive or zero. The D- and G- absolute deviations are compared for the equiradial designs and central composite designs having same axial or radial distance.

Table 5: D- and G-Absolute Deviations for Equiradial and Central Composite Designs

\begin{tabular}{|c|c|c|c|c|c|c|c|c|c|}
\hline \multicolumn{5}{|c|}{ Axial or Radial distance $(\alpha=1.0)$} & \multicolumn{5}{|c|}{ Axial or Radial distance $(\alpha=1.414)$} \\
\hline $\begin{array}{l}\text { Design size } \\
\mathrm{N}\end{array}$ & $\begin{array}{l}\text { Radial } \\
\text { point } \\
\mathrm{n}_{1}\end{array}$ & $\begin{array}{l}\text { Center } \\
\text { point } \\
\mathrm{n}_{\mathrm{c}}\end{array}$ & $\begin{array}{l}\text { D-absolute } \\
\text { deviation } \\
\text { D-AD }\end{array}$ & $\begin{array}{l}\text { G-absolute } \\
\text { deviation } \\
\text { G-AD }\end{array}$ & $\begin{array}{l}\text { Design size } \\
\mathrm{N}\end{array}$ & $\begin{array}{l}\text { Radial } \\
\text { point } \\
\mathrm{n}_{1}\end{array}$ & $\begin{array}{l}\text { Center } \\
\text { point } \\
\mathrm{n}_{\mathrm{c}}\end{array}$ & $\begin{array}{l}\text { D-absolute } \\
\text { deviation } \\
\text { D-AD }\end{array}$ & $\begin{array}{l}\text { G-absolute } \\
\text { deviation } \\
\text { G-AD }\end{array}$ \\
\hline 6 & 5 & 1 & 3.18 & 0 & 6 & 5 & 1 & 7.38 & 0 \\
\hline 7 & & 2 & 3.05 & 0 & 7 & & 2 & 7.10 & 0 \\
\hline 8 & & 3 & 2.86 & 0 & 8 & & 3 & 6.65 & 0 \\
\hline 9 & & 4 & 2.67 & 0 & 9 & & 4 & 6.20 & 0 \\
\hline 10 & & 5 & 2.50 & 0 & 10 & & 5 & 5.79 & 0 \\
\hline 7 & 6 & 1 & 2.49 & 0 & 7 & 6 & 1 & 5.49 & 0 \\
\hline 8 & & 2 & 2.45 & 10.44 & 8 & & 2 & 5.39 & 10.46 \\
\hline 9 & & 3 & 2.33 & 9.28 & 9 & & 3 & 5.13 & 9.29 \\
\hline 10 & & 4 & 2.20 & 8.35 & 10 & & 4 & 4.84 & 8.36 \\
\hline 11 & & 5 & 2.07 & 7.59 & 11 & & 5 & 4.57 & 7.60 \\
\hline 8 & 7 & 1 & 1.64 & 0 & 8 & 7 & 1 & 3.25 & 0.05 \\
\hline 9 & & 2 & 1.64 & 15.45 & 9 & & 2 & 3.31 & 15.53 \\
\hline 10 & & 3 & 1.67 & 14.68 & 10 & & 3 & 3.18 & 14.51 \\
\hline 11 & & 4 & 1.50 & 13.35 & 11 & & 4 & 3.04 & 12.71 \\
\hline 12 & & 5 & 1.43 & 12.23 & 12 & & 5 & 2.90 & 12.09 \\
\hline 9 & 8 & 1 & 0.35 & 0.05 & 9 & 8 & 1 & 0 & 0 \\
\hline 10 & & 2 & 0.36 & 0.64 & 10 & & 2 & 0 & 0.09 \\
\hline 11 & & 3 & 0.35 & 0.56 & 11 & & 3 & 0 & 0.09 \\
\hline 12 & & 4 & 0.33 & 0.54 & 12 & & 4 & 0 & 0.08 \\
\hline 13 & & 5 & 0.32 & 0.50 & 13 & & 5 & 0 & 0.08 \\
\hline
\end{tabular}




\section{Discussion of results}

\subsection{Findings regarding the equiradial designs}

For each N-point equiradial design, the determinant value of information matrix increases for increasing axial distance. The maximum and minimum scaled variances of prediction remain constant, except for approximation error, for changing axial distances. As $\mathrm{n}_{1}$ increases, the determinant value of information matrix decreases when the equiradial design contains only one center point. For equiradial designs having $\mathrm{n}_{\mathrm{c}} \geq 1$ center point, the determinant value of the associated information matrix decreases for increasing $n_{c}$. This holds true irrespective of the axial distance. At $\mathrm{n}_{\mathrm{c}}=1$, the maximum scaled variance of prediction associated with equiradial designs exactly equals the number of design points, $\mathrm{N}$. However, for $\mathrm{n}_{\mathrm{c}}>1$, the maximum scaled variance of prediction increases as $\mathrm{n}_{\mathrm{c}}$ increases with a ratio $S P V_{\text {max }}: S P V_{\text {min }}$ being 1 at $\mathrm{N}=6, \mathrm{n}_{\mathrm{c}}=1$. This shows that the design is rotatable at $\mathrm{N}=6$ and $\mathrm{n}_{\mathrm{c}}=1$. As $\mathrm{n}_{1}$ increases, the determinant value of information matrix of the equiradial design increases for $\mathrm{n}_{\mathrm{c}}>1$. However as $\mathrm{n}_{1}$ increases, the maximum scaled variance of prediction increases at $n_{c}=1$ and decreases at $\mathrm{n}_{\mathrm{c}}>1$.

\subsection{Findings regarding the central composite designs}

For N-point central composite design, the determinant values of information matrices are maximized for increasing axial distances. On the other hand, the maximum and minimum scaled variances of prediction remain constant for changing axial distances, except for approximation error. As $n_{1}$ increases, the determinant value of information matrix increases for $n_{c} \geq 1$. Also, as $n_{1}$ and $n_{c}$ increase, the maximum scaled variance of prediction generally increases (except at $\mathrm{n}_{1}=8$ ). As regards the addition of center points, the determinant value of information matrix decreases for increasing center points and rotatability is attained at $\mathrm{N}=6, \mathrm{n}_{\mathrm{c}}=1$.

\subsection{Findings regarding the design efficiency}

The D-efficiency value associated with $\mathrm{N}$-point equiradial design at $\rho=1.414$ is higher than the D-efficiency value associated with the corresponding $\mathrm{N}$-point equiradial design at $\rho=1.0$. However, G-efficiency value remains constant for the two categories of design, except for approximation error. The D-efficiency values associated with $\mathrm{N}$-point circumscribed central composite designs at $\alpha=1.414$ are higher than the D-efficiency values associated with corresponding N-point inscribed central composite designs at $\alpha=1.0$. Also, G-efficiency values remain constant for the two categories of design, except for approximation error. For a fixed axial distance or radius, the D-efficiency value associated with $\mathrm{N}$-point equiradial design is higher than the D-efficiency value associated with the corresponding $\mathrm{N}$-point central composite design. Similarly for a fixed axial radius distance, the G-efficiency associated with N-point equiradial design is generally higher than the D-efficiency associated with the corresponding N-point central composite design. At $\mathrm{n}_{\mathrm{c}}=1$, D-efficiency associated with the equiradial design decreases as $\mathrm{N}$ increases but increases as $\mathrm{N}$ increases at fixed $n_{c}>1$. At $n_{c}=1$, G-efficiency associated with the equiradial design decreases as $\mathrm{N}$ increases but increases as $\mathrm{N}$ increases at fixed $n_{c}>1$.

For changing $\mathrm{n}_{\mathrm{c}}$ center points, D-efficiency of equiradial design deceases as $n_{c}$ increases. For fixed $n_{c}$ center points, D-efficiency associated with central composite design increases as $\mathrm{N}$ increases. For changing $\mathrm{n}_{\mathrm{c}}$ center points, D-efficiency associated with central composite design decreases as $n_{c}$ increases. Although, variations seem to exist with $\mathrm{n}_{1}=8$ axial points, the G-efficiency associated with central composite design generally decreases with increasing design size, $\mathrm{N}$, for fixed center point, $\mathrm{n}_{\mathrm{c}}$. The $\mathrm{D}$ - and G-absolute deviations present themselves as good measures of similarity of experimental designs. Their values are zero if the pairs of designs are identical. For the two cases of design types studied, the G-absolute deviation (G-AD) is zero at $n_{c}=1$ center point and decreases for increasing values of $n_{c}$. It can further be seen from the results that as the design size $\mathrm{N}$ increases, $\mathrm{D}$ absolute deviation (D-AD) decreases. It is conjecturally hoped that at some large design size, the equiradial design and the central composite design having the same axial or radial distance will coincide (be identical) in their properties.

\section{Conclusion}

The behaviours of the spherical equiradial designs and central composite designs have been examined for changing axial distances, changing design sizes and increased center points. Two new measures of similarity of experimental designs have been proposed for comparing experimental designs defined by some related properties. When two or more designs are identical in terms of D-efficiency property, the new measure called D-absolute deviation (D-AD) will be zero. Similarly, when two or more designs are identical in terms of G-efficiency property, the new measure called G-absolute deviation (G-AD) will be zero. The similarity of equiradial design to the central composite design, as measured by D-absolute deviation is stronger as the design size, $\mathrm{N}$, increases. Although numerical illustrations presented in Table 5 reveal interesting properties associated with the new measures of similarity of experimental designs, there is need for analytical verification and justification.

\section{References}

[1] Akram, M. (2002). Central composite designs robust to three missing observations. A Ph.D Thesis, The Islamic University, Bahawalpur.

[2] Antille, G. and Weinberg, A. (2000). A Study of D-optimal Designs Efficiency for Polynomial Regression. Department of Econometrics, University of Geneva. http://www.unige.ch/ses/metri/.

[3] Atkinson, A. C. and Donev, A. N. (1992). Optimum Experimental Designs, Oxford: Oxford University Press, (1992).

[4] Box, G. E. P. \& Wilson, K. B (1951). On the experimental attainment of optimum conditions. J. R. Stat. Soc., Ser B (13)1-45.

[5] Iwundu M. P. (2016a). Useful Numerical Statistics of Some Response Surface Methodology Designs; Journal of Mathematics Research. Vol. 8, No. 4, pg. 40-67. doi:10.5539/jmr.v8n4p40 URL: https://doi.org/10.5539/jmr.v8n4p40.

[6] Iwundu, M. P. (2016b). On the behaviour of second-order N-point equiradial designs under varying model parameters. International Journal of Statistics and Applications, Vol. 6. No. 5, pg. 276-292.

[7] Iwundu, M. P. and Jaja, E. I. (2017) Precision of full polynomial response surface designs on models with missing coefficients. International Journal of Advanced Statistics and Probability. Vol. 5 (1), pg. 32-36. https://doi.org/10.14419/ijasp.v5i1.7491.

[8] Iwundu, M. P. and Otaru, O. A. P. (2014). Imposing D-optimality criterion on the design regions of the Central Composite Designs (CCD). SCIENTIA AFRICANA Vol. 13, No. 1, pg.109-119.

[9] Khuri, A. I. and Cornel, J. A. (1996) Response Surface: Design and Analysis. Second Edition. Marcel Dekker, Inc. New York.

[10] Kinai, R. (2015). Effect of Dropout on the Efficiency of DsOptimal Designs for Linear Mixed Models. Master of Arts Thesis, University of Kansas.

[11] Myers, R. H., Montgomery, D. C. and Anderson-Cook, C. M. (2009) Response Surface Methodology: Process and Product Optimization using designed experiments. 3rd Edition. John Wiley \& Sons, Inc. New Jersey.

[12] Rady, E. A., Abd El-Monsef, M. M. E. and Seyam, M. M. (2009) Relationship among several optimality criteria. interstat.statjournals.net $>$ YEAR $>$ articles.

[13] Smucker, B. J., Jensen, W. Wu, Z. and Wang, B. (2016). Robustness of Classical and Optimal Designs to Missing Observations. Computational Statistics \& Data Analysis. 
http://doi.org/10.1016/j.csda.2016.12.001. Available online January 2017, pg. 1-17.

[14] Srisuradetchai, P. (2015). Robust response surface designs against missing observations. A Ph.D dissertation, Montana State University, Bozeman, Montana.

[15] Yakubu, Y., Chukwu, A. U., Adebayo, B. T. and Nwanzo, A. G. (2014). Effects of missing observations on predictive capability of central composite designs. International Journal on Computational Sciences \& Applications. Vol. 4, No. 6, pg. 1-18. DOI:10.5121/ijcsa.2014.4601 